REVIEW

\title{
Current status of paediatric heart, lung, and heart-lung transplantation
}

\author{
M Burch, P Aurora
}

Arch Dis Child 2004;89:386-389. doi: 10.1136/adc.2002.017186

Since the first successful heart transplantation by Christian Barnard in 1967, there have been over 60000 heart transplants performed worldwide. Around 350 paediatric heart transplants are now performed annually and approximately $10 \%$ of these are in the UK.

See end of article for authors' affiliations

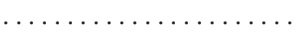

Correspondence to: Dr M Burch, Dept of Cardiology, Great Ormond Street Hospital for Children, London WC1N 3JH, UK;

Burch|M@gosh.nhs.uk

Accepted 29 June 2003

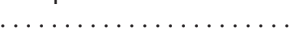

ung or heart-lung transplantation has been a therapeutic option for end stage lung disease - since the early 1980s, and there are approximately 1500 lung or heart-lung transplant procedures performed each year. As with heart transplants, the great majority of these procedures are performed in adults. Since the mid 1990s the numbers of paediatric lung and heartlung transplant procedures has stabilised at between 60 and 90 procedures performed annually worldwide, with the majority of these being performed in children with cystic fibrosis. ${ }^{1}$ In the UK, paediatric thoracic transplantation services have been concentrated in two centres: London (Great Ormond Street Hospital for Children) and Newcastle (Freeman Hospital). The London unit is the result of the merging of Great Ormond Street Hospital for Children with Harefield Hospital, thereby establishing it as one of the largest paediatric heart transplant programmes in the world. In some adult centres, there are now more lung and heart-lung transplants performed per annum than heart transplants. In most paediatric centres, including our own, heart transplants form the great majority of the caseload.

Despite the surgical team being common to both, there are really two distinct patient groups, the heart transplants being the domain of the cardiologist and the lung and heart-lung transplants patients best managed by respiratory paediatricians. One major difference between the two populations is the difficulty in obtaining donor organs. A recent report from our centre showed that less than half the paediatric cystic fibrosis patients listed between 1989 and 1999 received lung transplants, the remaining children having died on the waiting list. ${ }^{1}$ This situation is probably not improving, and this must be considered when referral for transplantation is being contemplated, as discussed in the second section of this article which deals with paediatric lung transplantation. In contrast, over the past two years, there have been no deaths on the Great Ormond Street heart transplant list. Urgent listing and the use of marginal, often adult, donors has helped critically ill children obtain a heart within three weeks over the past two years and facilitates mechanical support. There are fewer donors available for infants, and although this shortage has been improved by the use of mismatched blood group transplants, it remains a significant problem and small children can have very long waiting times. The match of recipient need and donor availability has been improved by the recent advances in the treatment of end stage heart failure. The outcome after heart transplantation is also improving (fig 1). These issues are discussed in the first section of this review, which deals with cardiac transplantation.

\section{HEART TRANSPLANTATION}

There have been some important new developments in paediatric heart transplantation in recent years. The advances discussed below are those that have had the greatest impact on our practice.

\section{Infant transplantation and $\mathrm{ABO}$ mismatch} Infants account for around $20 \%$ of paediatric heart transplants worldwide. The number of suitable donors is limited. ${ }^{2}$ Recently West et al from the Hospital for Sick Children, Toronto, reported the use of $\mathrm{ABO}$ incompatible heart transplantation in 10 infants. ${ }^{3}$ Usually transplantation of $\mathrm{ABO}$ incompatible donors is contraindicated because of the risk of hyperacute rejection from antibodies to the blood group antigens of the donor. This does not appear to apply to infants who have not yet produced isohaemagglutinins, and serum anti-A and anti$\mathrm{B}$ antibody titres are usually low in the first year of life. The upper age limit at which a mismatch can be given has not been determined and probably depends on the level of isohaemagglutinins present. If a significant level is present pretransplantation, it would be unwise to proceed. There have been five $\mathrm{ABO}$ mismatch transplants performed at Great Ormond Street and we have had no deaths at follow up so far. Post-transplant isohaemagglutinin levels are monitored at follow up and so far there have been no significant increases. Biopsy specimens are also examined for complement and antibody as well as the usual histology. All of this is extremely encouraging and does widen the pool of available donors.

\section{Advances in the management of heart failure}

The COCPIT study in adults ${ }^{4}$ showed that only patients with severe heart failure had a survival benefit from transplantation, which has major implications for heart transplantation. The study 


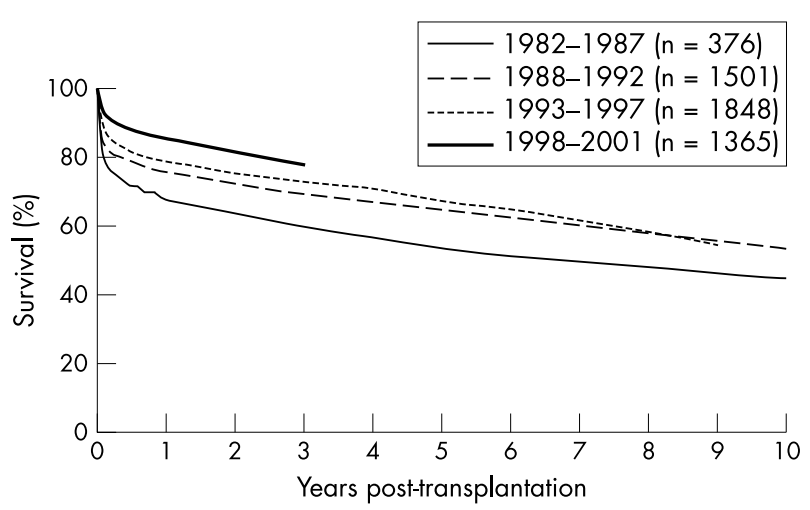

All comparisons with 1998-2001 are significant at $p<0.0001$ Comparison of 1982-1987 v 1988-1992 was significant at $p=0.003$ Comparison of 1982-1987 v 1993-1997 was significant at $p<0.0001$

Figure 1 Survival for paediatric heart transplantation by era. Reproduced from the International Society of Heart and Lung Transplantation at www. ishlt.org. ${ }^{27}$

has been criticised for the short observation period and incomplete data, and the clinical outcomes after transplant may be different in other units. It is however, widely accepted that the prognosis for heart failure in adults is improving and the new treatments for adult heart failure are beginning to permeate into paediatrics. At Great Ormond Street we have established a heart failure clinic, which facilitates the introduction of new therapies and allows a progression to transplantation assessment if necessary. We have found the use of maximal oxygen consumption helpful in assessment of severity of heart failure, and it can be used in the timing of paediatric heart transplantation referral, as it has been in adults. A value of $14 \mathrm{ml} / \mathrm{kg} / \mathrm{min}$ has been shown to be of prognostic use. ${ }^{5}$ The ability to use the exercise bicycle depends on age and height. It is not suitable for preschool children.

The use of angiotensin converting enzyme inhibitors and $\beta$ blockers has improved survival in heart failure. ${ }^{67}$ Carvedilol, a $\beta$ blocker with vasodilating properties, has been shown to improve outcome even when used in quite severe heart failure. ${ }^{7}$ We have used carvedilol in 30 children with heart failure; all were on angiotensin converting enzyme inhibitors and approximately half had echocardiographic fractional shortening of $<10 \%$. Although half had been on inotropes previously, it is clear from our experience and the literature that carvedilol must be used cautiously, if at all, in any patient very recently treated with inotropes or with signs of gross congestive failure. Cardiac resynchronisation therapy has been successful in adults with heart failure. ${ }^{8}$ We have used it in three children, including one infant in whom the improvement was encouraging.

\section{Mechanical support}

The advances in heart failure management and the COCPIT study both outlined above have contributed to a tendency for more children to be at UNOS (United Network for Organ Sharing) category $\mathrm{l}$ at transplant; either $\mathrm{lb}$, that is, requiring inotropes or increasingly, la which is requiring high dose inotropes or mechanical assistance. In the past there were few programmes that were able to bridge children to transplantation with mechanical support. North American centres have reported success with ECMO (extracorporeal membrane oxygenation) bridging. " This is not a long term support, but is an option for several weeks. At Great Ormond Street we have bridged 12 children for transplantation using ECMO, and there were no deaths at transplant. This policy is not without complications, we have had a recent problem with severe intracranial bleeding in a small child after three weeks of ECMO begun during prolonged cardiac arrest. ECMO bridging to transplant does require the increased use of marginal donor hearts, and approximately half of our heart transplant donors could be described as marginal with, for example, one of the following: a size mismatch of over $3 \times$ recipient body weight, high inotrope requirement of the donor, and longer ischaemic time. The morbidity of ECMO is substantial, often tracheostomy is required, there is commonly renal dysfunction requiring haemofiltration, and muscle weakness postoperatively leads to delayed mobilisation. ECMO is however, attractive to units such as ours as the nursing and surgical skill needed is already present. The neck cannulation can be done quickly and with low morbidity. Our low mortality with ECMO bridging does make us reluctant to change until there is compelling evidence of success with other devices. In our experience the alternative mechanical devices do seem to have a more difficult perioperative course with more bleeding and mechanical failure, but improvements are being made in this area, and other centres in Europe have made significant advances. ${ }^{10}$ Certainly, the shortage of donors for younger children and infants makes bridging of these children with ECMO difficult, because the wait for transplantation is likely to be months rather than weeks in Europe and ECMO is not likely to bridge successfully for such long periods. At present we do not use ECMO to bridge infants to transplantation, but would use a short period of ECMO to bridge to recovery. In the future it is likely that more permanent mechanical bridges to transplantation will be available, making it possible to discharge children home and wait for a suitable donor.

\section{Survival after heart transplantation}

When compared to paediatric heart transplantation a decade ago there is now an obvious improvement in surgical/30 day mortality (fig 1). There have been no 30 day deaths in our programme over the last year. This would compare to a perioperative mortality in excess of $20 \%$ a decade ago. This advance is largely the result of a great leap forward in paediatric intensive care. An important aspect of the latter is the use of ECMO to support failing hearts postoperatively, and we have used this four times in the past two years. Immune suppression is also more effective, and new drugs such as tacrolimus and mycophenolate are becoming more widely used. Tacrolimus is now our first line calcineurin inhibitor, rather than ciclosporin; it is more effective in preventing acute rejection in liver and kidney transplants. ${ }^{11}{ }^{12}$ Steroids are also used less commonly now; this is associated with reduced hypertension and obesity, and reduced coronary disease. The latter is also associated with acute rejection episodes and the improvements in immune suppression may have a substantial impact on this. The use of statins in reducing coronary disease has been encouraging; ${ }^{.13}$ surprisingly statins also appear to reduce the incidence of acute rejection, which may contribute to the improved survival associated with their introduction. We routinely use pravastatin now. The International Registry has shown improved survival by era (fig 1) and actuarial survival is approaching $80 \%$ at three years for the recent era.

\section{LUNG TRANSPLANTATION: BACKGROUND, ASSESSMENT, AND OUTCOME}

Our recommendations for referral and assessment for lung transplantation have recently been published, and we here present only a summary of our previous advice. ${ }^{14}$ 


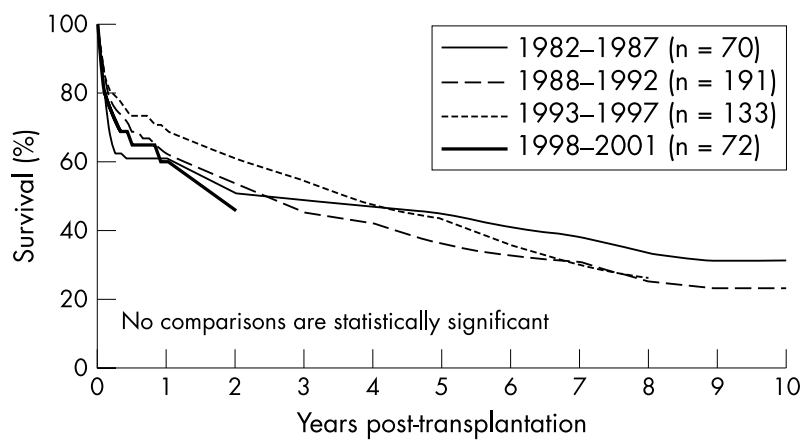

Figure 2 Survival for paediatric lung transplantation by era. Reproduced from the International Society of Heart and Lung Transplantation at www. ishlt.org. ${ }^{27}$

\section{Lung or heart-lung transplantation}

The majority of transplants performed in the 1980s were heart-lung transplants, where both heart and lungs are transplanted while the recipient is on cardiopulmonary bypass. The explanted heart can then be transplanted into another patient with terminal cardiac disease (the domino procedure). Over the past 10 years there has been a worldwide shift away from heart-lung transplantation in favour of double or bilateral lung transplantation. This procedure either involves removal of both diseased lungs and transplant of both donor lungs during cardiopulmonary bypass (double lung transplantation), or removal and transplant of one lung followed by removal and transplant of the second lung (bilateral sequential lung transplantation). This latter procedure is increasingly favoured, as it does not normally require cardiopulmonary bypass. Both double and bilateral lung transplantation allow the donor heart to be transplanted to another recipient.

Living donor bilateral lobar transplantation was first described in 1994, and involves the removal of a left and right lower lobe from two volunteer donors and sequential transplant into the recipient. This technique overcomes problems of donor shortage. However there are difficult ethical issues involved, as the procedure is not without risk to the donors, and true informed consent in such emotionally charged situations may be difficult to establish. This technique is only currently available for adult recipients in the UK, on a trial basis.

\section{Outcome following transplantation}

Choice between lung or heart-lung transplantation is dependent on surgical and logistic considerations, and some centres believe that the latter procedure results in fewer problems in younger children. The results for the two procedures are similar, and are poorer than for other solid organ transplants, with the International Society for Heart and Lung Transplantation reporting post-transplant survival of around $75 \%$ at one year and around $40 \%$ at five years (figs 2 and 3) for both procedures (www.ishlt.org). A recent analysis of our own results suggests that outcome at our centre is steadily improving, with five year survival having increased from $27 \% 10$ years ago, to $57 \%$ in the most recent era (authors' own data). This improvement in outcome with era is not mirrored in pooled international data, and we are unable to give an explanation for this.

The relatively poor outcome is related to the susceptibility of the transplanted lung to graft rejection, with the majority of the early deaths related to acute rejection or to overwhelming infection as the patient is heavily immunosuppressed. Those patients who survive the early post-transplant period are still at high risk of developing bronchiolitis obliterans syndrome (BOS). This condition is incompletely

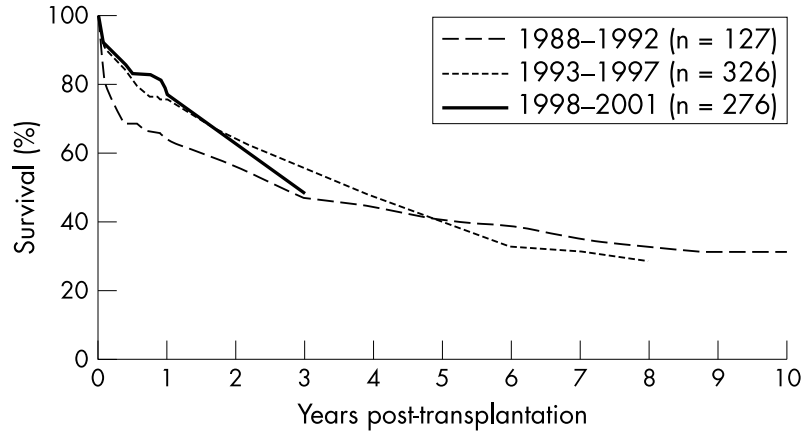

Figure 3 Survival for paediatric heart lung transplantation by era. Reproduced from the International Society of Heart and Lung Transplantation at www.ishlt.org. ${ }^{27}$

understood, and although it may be a form of chronic lung rejection, it is also related to episodes of acute rejection and lower respiratory infection in the early post-transplant period. ${ }^{15}{ }^{16}$ Once developed, it is irreversible, and is the major contributor to morbidity and mortality in patients surviving the first three months. Up to $50 \%$ of early survivors will develop BOS by three years post-transplant. ${ }^{16}$

\section{Selecting patients for transplantation}

With such limited post-transplant survival, the timing of transplantation has become an important clinical decision. The decision to recommend lung transplantation in children depends on three factors: life expectancy of two years or less; poor quality of life; and no contraindications to transplantation. The two former are discussed below. As with heart transplantation, there are few absolute contraindications to transplant and relative contraindications are regularly reviewed. It is always advisable to check with the transplant centre if there is concern about suitability. One recent development has been in our understanding of the impact of Burkholderia cepacia infection in CF subjects undergoing transplantation. Recently published data suggest that patients infected with $B$ cepacia genomovar III have an extremely poor post-transplant outcome, while infection with other cepacia genomovars has little effect on outcome. ${ }^{17}$

\section{Prediction of life expectancy}

There have now been a number of studies that have employed proportional hazards modelling in order to identify measurements that are of prognostic value in CF patients, with particular emphasis on the use of lung function measurements to determine life expectancy. ${ }^{18-22}$ The most recent study in children identified young age, female sex, low $\mathrm{FEV}_{1}$, low arterial oxygen saturation during the 12 minute walk test, high age adjusted resting heart rate, low plasma albumin concentration, and low blood haemoglobin concentration as the best predictors of survival in children with CF. The results from this study are in line with previous recommendations that children should be referred for transplantation assessment when their $\mathrm{FEV}_{1}$ falls to $30 \%$ or lower, but that younger children, girls, and patients deteriorating rapidly should be considered for referral sooner. The guidelines for referral of children with other respiratory diseases are very similar, but they are based on extrapolation from CF survival rather than on separate analyses.

\section{Quality of life}

Assessment of quality of life (QOL) is even less exact than estimation of life expectancy, as there are no widely accepted measures of QOL in childhood. Recent studies have attempted to objectively measure QOL in children with $\mathrm{CF}^{23}$ but as yet these scoring systems have not been applied 
to transplant assessment. In our centre, assessment is made by the clinician in combination with other members of the transplant team, and takes account of ability to partake in daily activities such as schooling or social activity, exercise tolerance, time spent in hospital, and requirement for oxygen and intravenous antibiotic therapy. As far as possible this assessment is taken from the child's perspective rather than from the parents, and detailed information from the referring centre is of great value. Although inexact, assessment of quality of life is an essential component of the risk assessment. A child with an $\mathrm{FEV}_{1}$ of $30 \%$ is unlikely to be accepted for transplantation if they still maintain a quality of life acceptable to that child.

\section{Benefits of lung transplantation}

There is increasing evidence that lung or heart-lung transplantation can increase life expectancy in appropriately selected patients. Testing this hypothesis has not been straightforward, as randomised controlled trials would not be acceptable in this situation. However, hazards modelling techniques can be employed to calculate the survival benefit from transplantation. There have now been a few published studies that have employed these methods and obtained similar results. ${ }^{14-26}$ The one study that has been performed in a paediatric population calculated a hazard ratio for transplantation of 0.31 , equating to a reduction in risk of death of $69 \%$ (95\% confidence interval $28-87 \%$ ). ${ }^{1}$

Assessing the impact of transplantation on health related quality of life is even more difficult, but there is some evidence that quality of life is enhanced in early survivors of lung transplantation. There are no published data from children, largely because of the difficulty in measuring quality of life in childhood. However, it is known that over $90 \%$ of children still surviving three years post lung transplantation have no limitation to their activity. Staff working within transplant programmes would insist that the majority of patients do have a substantial improvement in quality of life in the early post-transplant period, and it is this benefit, more than increase in longevity, that justifies the procedure.

\section{SUMMARY \\ Lung, heart-lung}

The initial enthusiasm for lung or heart-lung transplantation as therapy for end stage lung disease has been tempered, as outcome has remained poorer than for other solid organ transplants, and the supply of organs remained limited. For those children who do opt for transplantation, the majority can expect an increase in longevity with improved pulmonary function, allowing them to undertake previously impossible activities. It is the latter benefit, which in some children results in an extraordinary improvement in their quality of life, which ultimately justifies the procedure.

\section{Heart}

The results following heart transplantation remain significantly better than those following lung transplantation, substantial improvements in postoperative mortality have been achieved, and increased long term survival can be expected if coronary artery disease is reduced by newer treatment strategies. There are now around 200 children who are survivors of cardiac transplant in the UK, and more paediatricians are becoming involved in shared care of such patients. Heart transplantation is not a permanent cure, but for children with terminal cardiac failure it can be considered a life saving treatment, as it results in long survival with good quality of life for most recipients.

\section{Authors' affiliations}

M Burch, Dept of Cardiology, Great Ormond Street Hospital for Children, London, UK

P Aurora, Dept of Respiratory Medicine, Great Ormond Street Hospital for Children, London, UK

\section{REFERENCES}

1 Aurora P, Whitehead B, Wade A, et al. Lung transplantation and life extension in children with cystic fibrosis. Lancet 1999;354:1591-3.

2 Morrow WR, Naftel D, Chinnock R, et al. Outcome of listing for heart transplantation in infants younger than 6 months: predictors of death and interval to transplantation. J Heart Lung Transplant 1997; 16:1255-66.

3 West $\mathrm{U}$, Pollock-Barziv SM, Dipchand Al, et al. ABO-incompatible heart transplantation in infants. N Engl J Med 2001;344:793-800.

4 Deng MC, De Meester JM, Smits JM, et al. Effect of receiving a heart transplant: analysis of a national cohort entered on to a waiting list, stratified by heart failure severity. Comparative Outcome and Clinical Profiles in Transplantation (COCPIT) Study Group. BMJ 2000;321:540-5.

5 Mancini D, Le Jemtel T, Aaronson K. Peak $\mathrm{VO}_{2}$ : a simple yet enduring standard. Circulation 2000;101:1080-2.

6 The SOLVD Investigators. Effect of enalapril on survival in patients with reduced left ventricular ejection fractions and congestive heart failure. N Engl J Med 1991;325:293-302.

7 Packer M, Coats AJ, Fowler MB, et al. Effect of carvedilol on survival in severe chronic heart failure. N Engl J Med $2001 ; 344: 1651-8$.

8 Abraham WT, Fisher WG, Smith AL, and the MIRACLE Study Group. Cardiac resynchronization in chronic heart failure. N Engl J Med 2002;346:1845-53.

9 Gajarski RJ, Mosca RS, Ohye RG. Use of extracorporeal life support as a bridge to pediatric cardiac transplantation. J Heart Transplant 2003;22:28-34.

10 Stiller B, Dahnert I, Weng YG, et al. Children may survive severe myocarditis with prolonged use of biventricular assist devices. Heart 1999;82:237-40.

11 Margreiter R. Efficacy and safety of tacrolimus compared with ciclosporin microemulsion in renal transplantation: a randomized multicentre study. Lancet 2002:359:741-6.

12 O'Grady JG, Burroughs A, Hardy P, et al. Tacrolimus versus microemulsified ciclosporin in liver transplantation: the TML randomized controlled trial. Lancet 2002;360:1119-25.

13 Kobashigawa JA, Katznelson S, Laks $\mathrm{H}$, et al. Effect of pravastatin on outcomes after cardiac transplantation. N Engl J Med 1995;333:621-7.

14 Aurora P, Balfour-Lynn IM. Transplantation and end of life issues in cystic fibrosis. Paediatric Respiratory Reviews 2000;1:114-20.

15 Kroshus TJ, Kshettry VR, Savik K, et al. Risk factors for the development of bronchiolitis obliterans syndrome after lung transplantation. J Thoracic Cardiovasc Surg 1997; 1 14:195-202.

16 Sharples LD, Tamm M, McNeil K, et al. Development of bronchiolitis obliterans syndrome in recipients of heart-lung transplantation-early risk factors. Transplantation 1996;61:560-6.

17 De Soyza A, McDowell A, Archer L, et al. Burkholderia cepacia complex genomovars and pulmonary transplantation outcomes in patients with cystic fibrosis. Lancet 2001;358:1780-1.

18 Kerem E, Reisman J, Corey M, et al. Prediction of mortality in patients with cystic fibrosis. N Engl J Med 1992;326:1187-91.

19 Hayllar KM, Williams SGJ, Wise AE, et al. A prognostic model for the prediction of survival in cystic fibrosis. Thorax 1997;52:313-17.

20 Aurora P, Wade A, Whitmore P, et al. A model for predicting life expectancy of children with cystic fibrosis. Eur Respir J 2000;16:1056-60.

21 Liou TG, Adler FR, Fitzsimmons SC, et al. Predictive 5-year survivorship model of cystic fibrosis. Am J Epidemiol 2001;153:345-52.

22 Aurora P, Gassas A, Ehtisham S, et al. The effect of pre-lung transplant clinical status on post transplant survival of children with cystic fibrosis. Eur Respir $J$ 2000;16:1061-4.

23 Powers PM, Gerstle R, Lapey A. Adolescents with cystic fibrosis: family reports of adolescent health-related quality of life and forced expiratory volume in one second. Pediatrics 2001;107:E70.

24 Sharples L, Hathaway T, Dennis C, et al. Prognosis of patients with cystic fibrosis awaiting heart and lung transplantation. J Heart Lung Transplant 1993; 12:669-74.

25 Geertsma A, TenVergert EM, Bonsel GJ, et al. Does lung transplantation prolong life? A comparison of survival with and without transplantation. $J$ Heart Lung Transplant 1998;17:51 1-16.

26 Hosenpud JD, Bennett LE, Keck BM, et al. Effect of diagnosis on survival benefit of lung transplantation for end-stage lung disease. Lancet 1998;351:24-7.

27 Boucek MM, Edwards LB, Berkeley M, et al. The Registry of the International Society of Heart and Lung Transplantation: Fifth Official Pediatric Report 2001-2002. J Heart Lung Transplant 2002;21:827-40. 